Pacific Journal of Mathematics

LINEAR FUNCTIONAL AND ANALYTIC CONTINUATION 


\title{
LINEAR FUNCTIONALS AND ANALYTIC CONTINUATION PROBLEMS
}

\author{
Philip Davis and Henty Pollak
}

1. Introduction. According to the Weierstrass principle of analytic continuation, a single-valued analytic function is completely determined by its values in a neighborhood of a point $z_{0}$. These values often are given by a power series representation at $z_{0}$

$$
f(z)=\sum_{n=0}^{\infty} a_{n}\left(z-z_{0}\right)^{n}, \text { where } a_{n}=f^{(n)}(0) / n !
$$

and numerous classical theorems relate preassigned properties of the function $f(z)$ in the large to the sequence of coefficients $\left\{a_{n}\right\}$. It is frequently convenient to think of these results as associating to a given class of functions a subset of the space $A$ of formal sequences $\left\{a_{n}\right\}$. Since power series are especially pertinent to circles, it is natural that these theorems have dealt largely with subsets of functions analytic in circles. In order to study classes of functions which are analytic in an arbitrary domain $B$, it is no longer natural to use power series. It is, for example, far more relevant to use expansions in terms of a set $\left\{\phi_{n}(z)\right\}$ of functions which are complete and orthonormal over $B$ in some metric.

Such sets of orthonormal functions are sufficiently numerous so that we may require additional properties. In particular, suppose that an arbitrary region $B$ and a set of linear functionals $L_{0}, L_{1}, \ldots$, are given. It is then possible to find a set of functions $\left\{\phi_{n}^{*}(z)\right\}$ analytic in $B$ which are not only complete and orthonormal over $B$ with respect to an inner product $(f, g)_{B}$, but are simultaneously biorthonormal to a set of linear combinations of $\left\{L_{n}\right\}$. In terms of the set $\left\{\phi_{n}^{*}(z)\right\}$, a function $f(z)$ which is regular in $B$ (and of a certain class) possesses an expansion

$$
f(z)=\sum_{n=0}^{\infty} L_{n}^{*}(f) \phi_{n}^{*}(z),
$$

Received May 1, 1952.

Pacific J. Math. 3 (1953), 47-72 
with

$$
L_{n}^{*}\left(\phi_{m}^{*}\right)=\delta_{m n} \quad(m, n=0,1, \ldots)
$$

and

$$
L_{n}^{*}=\sum_{k=0}^{n} a_{n k} L_{k}
$$

The coefficients $L_{n}^{*}(f)$ in (1.2) may be written in the alternate, but equivalent, form

$$
L_{n}^{*}(f)=\left(f, \phi_{n}^{*}\right)_{B}
$$

The significance of the equality (1.5) lies in the fact that the functionals $L_{n}$ ( and hence $L_{n}^{*}$ ) may be defined over a wider class of analytic functions than is the inner product $(f, \mathrm{~g})_{B}$. The development of a general theory of orthonormal analytic systems from this point of view may be found in [11], of which the pertinent parts have been incorporated in $\$ 2$. This section also contains statements as to conditions under which the above results are valid. Earlier work in this direction is due to S. Bergman, who considered several special systems of orthogonal functions of the type dealt with here. (Cf. [2] for complete references.)

The above process is thus seen to have "two degrees of freedom," in that ( subject to fairly general conditions) we may select both $B$ and $L_{n}$ quite arbitrarily. For example, let there be given a region $B$, and the particular set of functionals

$$
L_{n}(f)=f^{(n)}\left(z_{0}\right) \quad\left(n=0,1, \ldots ; z_{0} \in B\right) .
$$

The resulting $\left\{L_{n}^{*}\right\}$ and $\left\{\phi_{n}^{*}(z)\right\}$ are then pertinent to the study of functions defined by the Taylor series (1.1), but continuable to $B$ and of finite norm $(f, f)_{B} \cdot$

The general theory may be carried out for a variety of inner products $(f, g)_{B}$. In this paper, however, we shall restrict our attention to the inner product

$$
(f, g)_{B}=\iint_{B} f \bar{g} d x d y \quad(z=x+i y) .
$$

The general theorems of $\$ 2$ will hold with other inner products leading to a Hilbert space; the particular importance of ( 1.7$)$ will appear in $\$ 4$. 
For a given complete set of bounded linear functionals $\left\{L_{n}\right\}$, the introduction of the complete orthonormal system $\left\{\phi_{n}^{*}(z)\right\}$ related to $\left\{L_{n}\right\}$ and $B$ enables us to characterize the function $f(z)$ in the large in terms of the functional values $L_{n}(f)(n=0,1, \cdots)$. In $\$ 3$ we apply these methods to the following problem: Given a region $B$, and $k$ fixed points on the boundary of $B$, derive necessary and sufficient conditions on the functional values $\left\{L_{n}(f)\right\}$ in order that a function analytic in $B$ possess singularities (of any kind) at these points. Letting these points become dense on the boundary, we obtain a set of conditions, each necessary, and in their totality sufficient, in order that $f$ have $B$ as its complete domain of existence. The conditions which we shall give are not of the usual Hadamard type, but are readily formulated in terms of linear transformations of the coefficient space $A$.

In $\$ 4$ these results are used to derive a "change of sign" theorem of the Fatou-Pólya type for arbitrary regions, while in $\$ 5$ we obtain a gap theorem for functions represented by an orthogonal expansion.

Our methods are of considerable interest in that they exhibit the power of orthogonal functions to answer a number of questions hitherto attacked by other means, and in that they allow us to extend to general regions theorems which are usually confined to circles. Finally, it should be pointed out that most of the present methods are applicable to the theory of functions of several complex variables; cf. [1].

2. Linear functionals and orthonormal systems. Let $B$ be a region lying in in the complex $z$-plane $(z=x+i y)$. By $L^{2}(B)$ we shall designate the set of functions which are regular and single-valued in $B$, with

$$
\|f\|_{B}=(f, f)^{1 / 2}<\infty
$$

where we have used the abbreviation

$$
(f, g)=\iint_{B} f \bar{g} d x d y .
$$

By $S$ we shall designate a fairly general set of analytic functions for which $L^{2}(B) \subset S$. Let there be given a set of linear functionals $\left\{L_{n}\right\}(n=0,1, \ldots)$, each of which is defined over $S$ and satisfies the following conditions:

(a) Each $L_{n}$ is bounded over $L^{2}(B)$.

(b) The set $\left\{L_{n}\right\}$ is independent; that is, for each $n(n=0,1, \ldots)$ there exist functions $f_{0}, f_{1}, \cdots, f_{n} \in L^{2}(B)$ such that the Gram determinant $\left|L_{i}\left(f_{j}\right)\right|$ 
satisfies

$$
\left|L_{i}\left(f_{j}\right)\right| \neq 0 \text {. }
$$

Condition (a) implies, of course, that within $L^{2}(B)$ each functional $L_{i}$ possesses an inner product representation. But the requirement that $L_{i}$ be defined over a wider class $S$ means, in effect, that we are in possession of an independent definition of $L_{i}$ which is applicable to all functions of $S$. Thus, for example, each $L_{i}$ may be a differential or a "point" operator of the usual kind met in interpolatory function-theory and applicable to all functions which are analytic in an appropriate region; or they may be given by an inner product rule in a Yilbert space which is wider than but totally unrelated to $L^{2}(B)$.

For the set of nonnegative integers $n$, let us set up the following set of minimum problems $Q_{n}$ : To determine that function $\phi_{n}(z)$ of class $L^{2}(B)$ which satisfies the conditions

$$
L_{0}\left(\phi_{n}\right)=L_{1}\left(\phi_{n}\right)=\cdots=L_{n-1}\left(\phi_{n}\right)=0, L_{n}\left(\phi_{n}\right)=1 \text {, }
$$

and which minimizes the integral $\left\|\phi_{n}\right\|_{B}$. The following theorem summarizes the situation (cf. [11]).

THEOREM 1. Under conditions (a) and (b), the problem $Q_{n}$ has, for each nonnegative integer $n$, a unique solution $\phi_{n}(z)$ of class $L^{2}(B)$. The set of minimal functions $\left\{\phi_{n}(z)\right\}$ is orthogonal over $B$. The set is complete in $L^{2}(B)$ if and only if the set $\left\{L_{n}\right\}$ is complete in $L^{2}(B)$, that is, if and only if

$$
L_{n}(f)=0(n=0,1, \cdots), f \in L^{2}(B)
$$

implies that $f$ vanishes identically.

We shall designate the normalized functions $\phi_{n}$ by $\phi_{n}^{*}$. Thus we have

$$
\phi_{n}^{*}(z)=\phi_{n}(z) / k_{n} \quad(n=0,1, \ldots) \text {, }
$$

where

$$
k_{n}=\left\|\phi_{n}\right\|_{B}=\left[L_{n}\left(\phi_{n}^{*}(z)\right)\right]^{-1}
$$

It can be shown that the orthonormal set $\left\{\phi_{n}^{*}(z)\right\}$ can be obtained by taking the set of functions

$$
h_{n}(w)=\overline{L_{n, z} K_{B}(z, \bar{w})} \quad(n=0,1, \cdots)
$$


and orthonormalizing them according to the Gram-Schmidt process of orthogonalization. Here $K_{B}(z, \bar{w})$ designates the Bergman analytic kernel function of the region $B$ (cf. [2]), while the subscript $z$ in $L_{n, z}$ indicates that the functional operation $L_{n}$ is to be carried out on $K_{B}$ considered as an analytic function of the complex variable $z$. It is clear that explicit determinantal expressions for $\phi_{n}^{*}(z)$ and $k_{n}$ can be derived; but inasmuch as we shall not require a knowledge of their precise structure, it will not be necessary to display them.

Let us next introduce a set of linear functionals $\left\{L_{n}^{*}\right\}$ which are related to $L_{n}$ by finite linear combiration, as follows:

$(2.8 \mathrm{a}) L_{n}^{*}(f) \equiv\left|\begin{array}{ccccc}L_{0}\left(\phi_{0}^{*}\right) & 0 & 0 & \cdots L_{0}(f) \\ L_{1}\left(\phi_{0}^{*}\right) & L_{1}\left(\phi_{1}^{*}\right) & 0 & \cdots L_{1}(f) \\ \cdot & \cdot & \cdot & \cdots & \cdot \\ \cdot & \cdot & \cdot & \cdots & \cdot \\ \cdot & \cdot & \cdots & \cdots \\ L_{n}\left(\phi_{0}^{*}\right) & L_{n}\left(\phi_{1}^{*}\right) & L_{n}\left(\phi_{2}^{*}\right) \cdots L_{n}(f)\end{array}\right| \cdot k_{1} \cdots k_{n} k$

for $n=1,2, \cdots$, and

$$
i_{0}^{*}(f)=k_{0} L_{0}(f) .
$$

It is clear that we may write

$$
L_{n}^{*}=\sum_{k=0}^{n} a_{n k} L_{k} \text {, }
$$

where the constants $a_{n k}$ are, essentially, the cofactors the last column of (2.8a). The constants $a_{n k}$ may be obtained explicitly from the values

$$
L_{m, w} \overline{L_{n, z} K_{B}(z, \bar{w})} \quad(m, n=0,1, \cdots),
$$

and the values of the inner products

$$
\overline{\left(L_{n, z} K_{B}(z, \bar{w})\right.}, \overline{\left.L_{m, z} K_{B}(z, \bar{w})\right)} \quad(m, n=0,1, \ldots,)
$$

by elementary operations.

Inasmuch as the functionals $z_{n}^{\prime}$ have been obtained by finite linear combination, it is clear that they are also defined over all functions of the larger class $S$. The principal facts about $\left\{L_{n}^{*}\right\}$ are as follows. For all $f \in L^{2}(B)$, we have 


$$
L_{n}^{*}(f)=\left(f, \phi_{n}^{*}\right) \quad(n=0,1, \cdots) .
$$

In particular, then, the sets $\left\{\phi_{n}^{*}\right\}$ and $\left\{L_{n}^{*}\right\}$ are biorthonormal in the sense that

$$
L_{m}^{*}\left(\phi_{n}^{*}\right)=\delta_{m n} \quad(m, n=0,1, \cdots) .
$$

The functions $\dot{\phi}_{n}^{*}$ may therefore be considered as a doubly orthogonal set, orthogonal once in the sense of the inner product (2.2) and once in the sense of (2.11).

When the set $\left\{\phi_{n}^{*}(z)\right\}$ (or, what is equivalent, $\left\{L_{n}\right\}$ ) is complete for $L^{2}(B)$, the Fourier expansion of a function $f \in L^{2}(B)$ may be written as

$$
f(z)=\sum_{n=0}^{\infty}\left(f, \phi_{n}^{*}\right) \phi_{n}^{*}(z),
$$

or in the equivalent form

$$
f(z)=\sum_{n=0}^{\infty} L_{n}^{*}(f) \phi_{n}^{*}(z) .
$$

It should be pointed out that a series (2.13) may be defined for all functions $f$ of the wider class $S$. In some cases (depending upon $\left\{L_{n}\right\}$ ) it has even been possible to show [11] that the series (2.13) will represent $f$ even if $f$ is regular only in a subregion of $B$. The following theorem is now' a consequence of the foregoing remarks.

THEOREM 2. Let there be given a set of linear functionals $\left\{L_{n}\right\}$ satisfying (a) and (b) and defined over a linear class $S$ of functions where $S$ is larger than $L^{2}(B)$. Suppose, furthermore, that $\left\{L_{n}\right\}$ is complete with respect to $S$. Then a given $f \in S$ is of class $L^{2}(B)$ if and only if

$$
\sum_{n=0}^{\infty}\left|L_{n}^{*}(f)\right|^{2}<\infty .
$$

We shall give here a brief indication of the proof of this theorem. Assume that $f \in L^{2}(B)$. Then by (2.10) and the Riesz-Fischer theorem (cf. [2]) for the space $L^{2}(B),(2.14)$ must hold. Conversely, let there be given an $f \in S$ with (2.14) holding, and construct the function 


$$
g(z)=\sum_{n=0}^{\infty} L_{n}^{*}(f) \phi_{n}^{*}(z)
$$

By the Riesz-Fischer theorem we have $g(z) \in L^{2}(\delta)$. From (a) and (2.11) we obtain

$$
L_{n}^{*}(f)=L_{n}^{*}(g)
$$

and therefore

$$
L_{n}^{*}(f-g)=0 \quad(n=0,1, \ldots) .
$$

Yience, by our assumption that $\left\{L_{n}\right\}$ is complete in $S$, it follows that $f \equiv g$.

Let us make here an observation which will be of use in the sequel. Suppose that all the hypotheses of Theorem 2 are fulfilled except that no assumption is made with respect to the completeness of $\left\{L_{n}^{*}\right\}$. Then, if for some $f \in S$ we have

$$
\sum_{n=0}^{\infty}\left|L_{n}^{*}(f)\right|^{2}=\infty
$$

it follows from the Riesz-Fischer theorem that $f \notin L^{2}(B)$.

It should be remarked that the set $\left\{\phi_{n}^{*}(z)\right\}$ depends both upon the domain $B$ and upon the preassigned set of functionals $\left\{L_{n}\right\}$; when we wish to emphasize this fact we shall write $\phi_{n}^{*}\left(z, B ;\left\{L_{j}\right\}\right)$, designating thereby the unique functions constructed in the manner previously described.

A further result taken from [11], and which we shall find of use in the sequel, is the following. Let $B$ be a bounded region containing the origin such that the boundary of $B$ consists of a finite number of mutually disjoint Jordan curves. Let there be given a sequence of points $\left\{\alpha_{n}\right\}$ lying in $B$ for which ${ }^{1}$

$$
\lim _{n \rightarrow \infty} \alpha_{n}=0
$$

Introduce the set of functionals $\left\{L_{n}\right\}$, defined by

$$
L_{n}(f)=f\left(\alpha_{n}\right) \text {, }
$$

and construct the orthogonal functions

\footnotetext{
${ }^{1}$ Condition (2.16) may be generalized.
} 


$$
\phi_{n}^{*}(z)=\phi_{n}^{*}\left(z ; B,\left\{L_{j}\right\}\right)
$$

If now $f(z)$ is analytic in $B$, then the interpolation series

$$
f(z) \sim \sum_{n=0}^{\infty} L_{n}^{*}(f) \phi_{n}^{*}(z)
$$

converges to $f(z)$ uniformly in any closed subset of $B$. Furthermore, we have

$$
\limsup _{n \rightarrow \infty}\left|L_{n}^{*}(f)\right|^{1 / n} \leq 1
$$

Conversely, if a series (2.18) is given for which (2.19) holds, then the sum of the series is analytic throughout $B$, and the function represented has the given series $(2.18)$ as its formal expansion found by interpolation in the points $z=\alpha_{i}$. It should be observed that when $f \in L^{2}(B)$ we have, by (2.10),

$$
L_{n}^{*}(f)=\iint_{B} f \bar{\phi}_{n}^{*} d x d y \quad(n=0,1, \cdots) .
$$

A corresponding result holds when the points $a_{n}$ coincide at $z=0$. In this case, the functionals $L_{n}$ are to be interpreted as

$$
L_{n}(f)=f^{(n)}(0) \quad(n=0,1, \ldots) .
$$

For complete details the reader is referred to [11].

Theorem 2 is the point of departure for the investigations of this paper. Developments analogous to those presented here are valid for classes of functions other than $L^{2}(B)$. In particular, they are valid for functions of several complex variables. In this case, we deal with the space $L^{2}\left(B^{(2 n)}\right)$ of single-valued analytic functions of $n$ complex variables $f\left(z_{1}, \ldots, z_{n}\right)$ for which

$$
\begin{array}{r}
\int_{B}(2 n) \\
|f|^{2} d w<\infty ; \\
d w=d x_{1} d y_{1} \cdots d x_{n} d y_{n} \\
\left(z_{j}=x_{j}+i y_{j} ; j=1,2, \cdots, n\right) .
\end{array}
$$

Generalizations to nonschlicht domains in the space of one or several complex variables can also be effected. The contents of Theorem 2 can be given a more abstract formulation, but we shall not do so here inasmuch as our principal concern is with its application to analytic function theory.

3. Criteria for analytic continuation. At the outset we shall introduce the 
following notation. The set of all sequences of complex numbers $\left\{a_{n}\right\}$ shall be designated by $A$. The sequential Hilbert space, that is, the subset of $A$ for which

$$
\sum_{n=0}^{\infty}\left|a_{n}\right|^{2}<\infty
$$

will be designated by $H^{2}$. Given the sequence $a \equiv\left\{a_{n}\right\}$, we shall designate by $b=T a$ the sequence $b \equiv\left\{b_{n}\right\}$ obtained from $a$ by means of a linear triangular transformation of the form

$$
b_{n}=\sum_{k=0}^{n} c_{n k} a_{k} \text {. }
$$

Our approach to the problem of analytic continuation can be described in the following general way. Let there be given an extensive class of analytic functions $S$ and a set $\left\{L_{n}\right\}$ of linear functionals which is complete in $S$. In addition, let there be given a distinguished subset $S^{*}$ of $S$. It will be supposed that the distinguished subset $S^{*}$ can be characterized geometrically as follows. We shall suppose that there exists one [and possibly two $]^{2}$ sequences of regions $\left\{B_{n}\right\}$ [ and $\left.\left\{C_{n}\right\}\right]$ such that $L^{2}\left(B_{n}\right) \subset S\left[\right.$ and $L^{2}\left(C_{n}\right) \subset S$ ] while $f \in S^{*}$ if and only if

$$
f \in L^{2}\left(B_{n}\right) \quad(n=0,1, \ldots)
$$

[ and

$$
f \notin L^{2}\left(C_{n}\right)
$$$$
(n=0,1, \ldots)]
$$

Furthermore, we make the assumption that each functional $L_{n}$ satisfies conditions (a) and (b) with respect to all the regions $B_{j}$ [ and $\left.C_{j}\right]$. Let there now be given an $f \in S^{*}$. This function is determined in a unique way within $S$ by the values $L_{n}(f)(n=0,1, \ldots)$. We may therefore ask for a criterion phrased in terms of the values $L_{n}(f)$ which will tell us whether or not $f$ is, in addition, a member of the distinguished subclass $S^{*}$. Such a criterion now follows from (3.2) and Theorem 2. For each region $B_{j}\left[\right.$ and $\left.C_{k}\right]$, let us construct the orthonormal systems

$$
\phi_{n, j}^{*}=\phi_{n, j}^{*}\left(z, B_{j},\left\{L_{m}\right\}\right)\left[\text { and } \phi_{n, k}^{*}=\phi_{n, k}^{*}\left(z, C_{k},\left\{L_{n}\right\}\right)\right] .
$$

\footnotetext{
${ }^{2}$ In dealing with certain classes $S^{*}$ we may need to include the conditions and construction given in brackets throughout the remainder of this paragraph. Cf. the examples which follow immediately.
} 
By means of (2.8) these sets of orthonormal systems, we then construct the related functionals $\left\{L_{n, j}^{*}\right\}\left[\right.$ and $\left.\left\{L_{n, k}^{*}\right\}\right]$. A necessary and sufficient condition that $f \in S^{*}$ is that

$$
\sum_{n=0}^{\infty}\left|L_{n, j}^{*}(f)\right|^{2}<\infty \quad(j=0,1, \ldots)
$$

[ and

$$
\left.\sum_{n=0}^{\infty}\left|L_{n, k}^{*}(f)\right|^{2}=\infty \quad(k=0,1, \ldots)\right]
$$

Inasmuch as the $L_{n}^{*}$, s are linear combinations of the functionals $L_{n}$, we may say that there exists one [and possibly two] sequences of transformations $\left\{T_{j}{ }^{(1)}\right\}$ [ and $\left.\left\{T_{j}^{(2)}\right\}\right]$ such that

$$
T_{j}{ }^{(1)}\left\{L_{n}(f)\right\} \in H^{2} \quad(j=0,1, \cdots)
$$

[ and

$$
T_{j}{ }^{(2)}\left\{L_{n}(f)\right\} \notin H^{2}
$$$$
(j=0,1, \cdots)]
$$

are necessary and sufficient conditions that $f \in S^{*}$. Equations (3.4) and (3.4') may be employed to study the topology of the set $S^{*}$ in the coefficient space $A$, and therefore the question as to what distinguished classes $S^{*}$ possess criteria of the type (3.4) is an interesting one which deserves attention. We shall now give a number of examples which will elucidate these general remarks.

EXAMPLE 1. Let $S$ be the class of functions which are regular at $z=0$, and let $L_{n}(f)=f^{(n)}(0)(n=0,1, \ldots)$. Let $S^{*}$ be the class of functions which are regular and single-valued in the entire (finite) plane with the exception of $k$ ( single-valued) singularities located at the $k$ fixed points $z_{1}, \ldots, z_{k}\left(z_{i} \neq 0\right)$ which have been prescribed in advance. For the regions $C_{n}$, we may take $k$ regions, the $i^{\text {th }}$ of which contains the points $z=0$ and $z=z_{i}$. Let $r$ designate

$$
\max _{1 \leq i \leq k}\left|z_{i}\right|
$$

For $B_{n}$, take the circle $|z| \leq r+1+n$ from which has been deleted the $k$ smaller circles $\left|z-z_{i}\right| \leq 1 / n(i=1,2, \cdots, k)$. Suppose now that $f \in S^{*}$. Then it is clear that $f \in L^{2}\left(B_{n}\right)$ and $f \notin L^{2}\left(C_{n}\right)(n=0,1, \ldots)$. Conversely, if we have $f \in L^{2}\left(B_{n}\right)(n=0,1, \ldots)$, then surely $f$ is regular in the entire (finite) plane, 
with the possible exception of the points $z=z_{i}(i=1,2, \cdots, k)$, while $f \notin$ $L^{2}\left(C_{n}\right)(n=1,2, \cdots, k)$ ensures that singularities occur at these points.

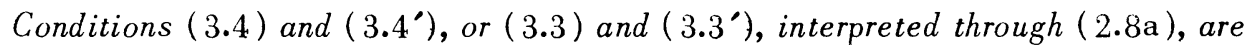
thus necessary and sufficient for the Taylor coefficients in order that the function be regular and single-valued in the finite plane with the exception of singularities at $k$ specified points.

Several remarks are now appropriate. It is clear, in the first place, that the sequences of regions $\left\{B_{n}\right\}$ and $\left\{C_{n}\right\}$ may be selected in a variety of ways, each selection yielding a necessary and sufficient condition. Secondly, the points at which the function is to have singularities need not be finite or even denumerably infinite, but may comprise quite general point sets. The criterion just given (and those given in subsequent examples) is, in principle, completely constructable. For, the kernel function $K_{B}(z, \bar{w})$ of a simply or multiply connected domain $B$ may be determined [2] through the orthonormalization of a set of functions complete for $L^{2}(B)$; the orthonormal functions $\phi_{n}^{*}$ may then be computed

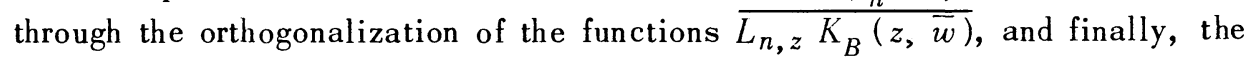
coefficients $a_{n k}$ in the expression

$$
L_{n}^{*}=\sum_{k=0}^{n} a_{n k} L_{k}
$$

may be computed through (2.8a). Thus, the sequences of coefficients which occur in $T_{j}{ }^{(1)}, T_{j}{ }^{(2)}$ may be completely specified beforehand, and are independent of the particular $f$ which is to be tested. Our criteria are, therefore, true necessary and sufficient conditions; but in most cases, because of their complexity, these conditions will be rather inaccessible for further analytic work. While the cases which yield further results are of prime importance, all cases are none the less of interest in so far as they show what classes of analytic functions $S^{*}$ can be characterized by transformations of the type considered in the coefficient space $A$.

EXAMPLE 2. Let $B$ be a region which contains the origin in its interior and is bounded by a finite number of nonintersecting Jordan curves. Let $S$ designate the class of functions regular at $z=0$. Let $S^{*}$ designate the class of functions which are regular in $B$ and possess $B$ as their total regions of existence; that is the boundary $b$ of $B$ is the natural boundary for each function of class $S^{*}$. Let us now construct two sequences of regions $B_{n}, C_{n}$ as follows. For $B_{n}$ take any sequence of regions whose closures lie entirely interior to $B$ and such that 


$$
B=\sum_{n=0}^{\infty} B_{n}
$$

Let now $z_{n}$ be a sequence of points which is dense on $b$. Let $V_{n}$ designate the circle $\left|z-z_{n}\right|<1 / n$, and set $C_{n}=B \cup V_{n}$. We may now show that $f(z)$ is of class $S^{*}$; that is, $f(z)$ is regular throughout $B$ and possesses the boundary of $B$ as a natural boundary if and only if

$$
\begin{array}{ll}
f \in L^{2}\left(B_{n}\right) & (n=0,1,2, \cdots), \\
f \notin L^{2}\left(C_{n}\right) & (n=0,1,2, \cdots) .
\end{array}
$$

For suppose, first of all, that $f \in S^{*}$. If $B^{\prime}$ is any subregion lying entirely in $B$, then it is clear that $f \in L^{2}\left(B^{\prime}\right)$, so that a fortiori (3.5a) holds. If $f \in L^{2}\left(C_{j}\right)$ for some $j, f$ would be regular in the region $B \cup V_{j}$ which is larger than $B$, and this would contradict the hypothesis that $f$ cannot be continued analytically to a larger region. Thus, (3.5b) is established. Conversely, let there be given an $f$ for which (3.5a) and (3.5b) hold. From (3.5a) and the fact that

$$
B=\sum_{n=0}^{\infty} B_{n}
$$

it is clear that $f$ must be regular in $B$. If now $f$ could be continued analytically across some portion of the boundary $b$, then there would exist a point $z^{\prime} \in b$ and a circle $V^{\prime}:\left|z-z^{\prime}\right|<r$ such that $f$ would be regular in $B \cup V^{\prime}$. Since the points $z_{n}$ are dense in $b$ and the radii of $V_{n}$ tend to 0 , we may surely find a point $z_{n} \in$ $b \cap V^{\prime}$ such that $V_{n} \subset V^{\prime}$. Then $C_{n}=B \cup V_{n}$ lies entirely interior to $B \cup V^{\prime}$, so that $f \in L^{2}\left(C_{n}\right)$. This is a contradiction, and therefore $f$ cannot be continued analytically across any portion of the boundary of $B$.

Let us now select

$$
L_{j}(f)=f^{(j)}(0)
$$$$
(j=0,1, \cdots) \text {, }
$$

and construct the functions $\phi_{n}^{*}\left(z, B_{n} ;\left\{L_{j}\right\}\right)$, the corresponding functions for the regions $C_{n}$, and the related functionals $L_{n, j}^{*}$. Then (3.3) and (3.3') are neces sary and sufficient conditions on the Taylor coefficients of a function $f$ in order that $f$ be regular in $B$ and possess the boundary of $B$ as a natural boundary.

If, in particular, $B$ is selected as the circle $|z|<1$, then our criterion gives necessary and sufficient conditions on the coefficients of 


$$
f(z)=\sum_{n=0}^{\infty} a_{n} z^{n}
$$

in order that $f$ possess $|z|=?$ as a natural boundary. In this special case, we may select as $z_{n}$, the points $z_{n}=e^{2 \pi i r_{n}}$ where $\left\{r_{n}\right\}$ designates the set of rationals $x$ for which $0 \leq x \leq 1$. The region $C_{n}$ is now the region composed of all points in $|z|<1$ and $\left|z-z_{n}\right|<1 / n$. $C_{n}$ may be mapped onto the interior of a circle by an elementary function $\phi_{n}(z)$ which is compounded of a löbius transformation plus a root extraction. Inasmuch as the kernel function $K_{C_{n}}(z, \bar{w})$ may be obobtained from $\phi_{n}(z)$ by means of the formula

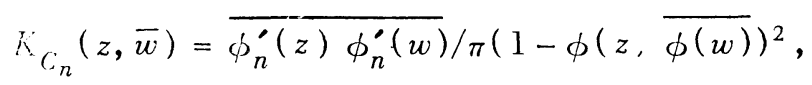

it is clear that $K_{C_{n}}(z, \bar{w})$, and hence the constants $a_{m, k}^{(n)}$ appearing in

$$
L_{m}^{*}\left(C_{n}\right)=\sum_{k=0}^{m} a_{m k}^{(n)} L_{k},
$$

may be computed in an elementary fashion. Despite this fact, it would seem that no very great simplification in the criteria given can be expected. The orthogonalization of the functions $\overline{L_{n, z} K(z, \bar{w})}$ which is met in (2.8) introduces considerable algebraic complexities. Let $N$ designate the class of analytic functions which possess $|z|=1$ as a natural boundary. That a necessary and sufficient condition for $f \in N$ ought to be fairly involved may be inferred from the fact that $N$ possesses no simple algebraic structure; given $f_{1}, f_{2} \in N$, then we cannot assert that any of the usual algebraic combinations $f_{1}+f_{2}, f_{1} f_{2}, f_{1} / f_{2}$, $f_{1}\left(f_{2}\right)\left[\right.$ here $\left.\left|f_{2}\right|<1\right]$ must of necessity belong to $N .^{3}$

For general regions $B$, the regions $B_{n}$ may be constructed by the usual device of covering $B$ by a grid. (See, for example, [9, p. 7-8], ) As regards the regions $C_{n}$, it suffices for applications that there exists a set $\left\{C_{n}\right\}$ satisfying the following conditions:

$$
B \subset C_{n}
$$$$
(n=0,1, \cdots),
$$

(d) if $B \subset B^{*}, B \neq B^{*}$, then there exists an index $j$ such that $C_{j} \subset B^{*}$.

We may then show by means of arguments similar to those just used that $f \in S^{*}$ if and only if $f \in L^{2}\left(B_{n}\right), f \notin L^{2}\left(C_{n}\right)(n=0,1, \ldots)$. We shall not examine the question as to what regions possess systems $\left\{C_{n}\right\}$ satisfying $(\mathrm{c})$ and $(\mathrm{d})$. In our applications we shall deal with explicitly constructed sets $\left\{C_{n}\right\}$.

\footnotetext{
${ }^{3}$ For a topological discussion of the set $N$ in the coefficient space $A$, see [8].
} 
We need not adhere to the selection $L_{n}(f)=f^{(n)}(0)$. As another interesting choice, let $G$ be a fixed region interior to $B$ and possessing a complete orthonormal set $\left\{r_{n}(z)\right\}$. Introduce

$$
L_{n}(f)=\iint_{B} f{\overline{\psi^{\prime}}}_{n} d x d y
$$

In this case, our criterion yields a necessary and sufficient condition on the Fourier coefficients of $f$ (with respect to $\left\{\psi_{n}\right\}$ ) that $f$ have $B$ as its natural region of existence.

EXAMPLE 3. Although the present example is not concerned with the question of analytic continuation, it provides an additional application of our methods. Considerable work has been done on functions which are bounded in the unit circle $|f(z)| \leq 1,|z|<1$. In particular, the coefficient problem for bounded functions, that is, the problem of determining the necessary and sufficient conditions on the coefficients of

$$
f(z)=\sum_{n=0}^{\infty} a_{n} z^{n}
$$

in order that $f$ be bounded in the unit circle, has been solved by I. Schur. (See, for example, $[3, \mathrm{p} .138-145])$. We turn our attention here to the corresponding problem for an arbitrary region, and show how Theorem 2 gives us an answer to this question.

At first we remark that Theorem 2 may be written in the following stronger form. Let there be given a set of linear functionals $\left\{L_{n}\right\}$ satisfying (a) and (b) and defined over a linear class of functions $S$ larger than $L^{2}(B)$. Suppose also that $\left\{L_{n}\right\}$ is complete with respect to $S$. Then a given $f \in S$ if of class $L^{2}(B)$ and possesses a norm

$$
\|f\|^{2}=\iint_{B}|f|^{2} d x d y=M^{2}
$$

if and only if

$$
\sum_{n=0}^{\infty}\left|L_{n}^{*}(f)\right|^{2}=H^{2}
$$

Let $B$ be a bounded region which is assumed to contain $z=0$ in its interior. 
It is easily shown that a necessary and sufficient condition that $|f(z)| \leq 1$ in $b$ is that

$$
\iint_{B^{\prime}}|f(z)|^{2} d x d y \leq \operatorname{area}\left(3^{\prime}\right)
$$

for all $B^{\prime} \subset B$.

It will be convenient for our purposes to obtain a condition of type (3.8) which utilizes only a sequence of subregions, each subregion containing the origin in its interior. (One way in which this can be accomplished is as follows. Let $\left\{z_{n}\right\}$ be a sequence of points which is dense in $B$. Let $C_{n}$ designate the circle

$$
\left|z-z_{n}\right|<\rho_{n}, \quad \rho_{n}=\min \left[\frac{1}{n}, \text { dist }\left(z_{n} \text { to boundary of } B\right)\right] \text {. }
$$

Since we are dealing with a connected set, it is possible to find an arc joining $z=0$ and $z=z_{n}$ and lying interior to $B$. It is therefore also possible to find a curvilinear strip $U_{n}$ issuing from $C_{n}$, lying interior to $B$, containing the origin in its interior, and such that

$$
\text { area }\left(U_{n}\right) \leqq \frac{1}{n} \text { area }\left(C_{n}\right) \quad(n=0,1, \ldots)
$$

Designate the simply connected region $C_{n} \cup U_{n}$ by $S_{n}$. We now show that a necessary and sufficient condition that $|f(z)| \leq 1$ in $B$ is that

$$
\iint_{S_{n}}|f(z)|^{2} d x d y \leq \text { area }\left(S_{n}\right) \quad(n=0,1,2, \ldots) .
$$

The condition obviously is necessary. Suppose, now, that (3.9) holds, while $\left|f\left(z^{\prime}\right)\right|>t>1$. By continuity, we have $|f(z)|>t$ in a neighborhood $N$ of $z^{\prime}$; hence, for an appropriate selection if indices $n_{k}(k=0,1, \cdots)$, we have also $|f(z)|>t$ throughout $C_{n_{k}}(k=0,1, \ldots)$. Now

$$
\frac{\iint_{S_{n_{k}}}|f(z)|^{2} d A}{\operatorname{area}\left(S_{n_{k}}\right)} \geq \frac{\iint_{C_{n_{k}}}|f(z)|^{2} d 1}{\left(1+1 / n_{k}\right) \text { area }\left(C_{n_{k}}\right)} \geq \frac{t}{1+1 n_{k}}>1
$$

for $k$ sufficiently large. This contradicts (3.9) and proves the statement.

Let

$$
L_{n}(f)=f^{(n)}(0) / n ! ;
$$


and let

$$
L_{n}^{*(k)}=\sum_{m=0}^{n} a_{n m}^{(k)} L_{m}
$$

be those functionals associated with $\left\{L_{n}\right\}$ and $\left\{S_{k}\right\}$ through (2.8). By (3.7), a necessary and sufficient condition that

$$
f(z)=\sum_{n=0}^{\infty} a_{n} z^{n}
$$

satisfy $|f(z)| \leq 1$ in $B$ is that

$$
\sum_{n=0}^{\infty}\left|\sum_{m=0}^{n} a_{n m}^{(k)} a_{m}\right|^{2} \leq \operatorname{area}\left(S_{k}\right) \quad(k=0,1 . \cdots)
$$

It is to be emphasized that the coefficients $a_{n m}^{(k)}$ depend only on $S_{k}$, and may therefore be computed in advance. Conditions (3.10) may be made the source of numerous inequalities to be satisfied by the individual coefficients.

4. Change-of-sign theorems. The following theorem was originally conjectured by Fatou [6]. Let

$$
f(z)=\sum_{n=0}^{\infty} a_{n} z^{n}
$$

possess the circle $|z|=1$ as a circle of convergence. Then there exists a sequence $\epsilon_{n}$ in which each $\epsilon_{n}$ is either +1 or -1 and such that the function

$$
f^{*}(z)=\sum_{n=0}^{\infty} \epsilon_{n} a_{n} z^{n}
$$

possesses $|z|=1$ as a natural boundary. The first proof of Fatou's conjecture is due to Pólya [7]. A frequently quoted proof (See for example, Bieberbach [3, vol. 2, p. 299-300].) is due to Hurwitz [7]. In the present section we shall show how certain generalizations of this theorem may be attacked by the method developed in Example 2 above.

In what follows, we shall assume that there has been given a region $B$ for which a sequence of regions $C_{n}$ satisfying properties (c) and (d) above may be 
found. $L_{y}\left\{L_{n}\right\}$ we shall designate a set of linear functionals which are defined over a class $S$ of function which is larger than $L^{2}(B)$. The set $\left\{L_{n}\right\}$ will be assumed complete with respect to $S$, and in addition, for each $f \in L^{2}(B)$ and for each $n$, it will be assumed that we have a representation of the form

$$
L_{n}(f)=\iint_{B} f \overline{p_{n}} d x d y \quad(n=0,1, \cdots),
$$

where $p_{n}(z) \in L^{2}(\xi)(n=0,1, \ldots)$, and $\left\{p_{n}(z)\right\}$ is a complete and orthonornal set for $L^{2}(B)$. Thus, the quantities $L_{n}(f)$, for $f \in S$ may be regarded as generalized Fourier coefficients of $f$ with respect to the orthonormal set $\left\{p_{n}(z)\right\}$.

We shall now mention several examples of such sets of functionals.

(1) Let $B$ be such that $L^{2}(B)$ possesses a complete orthonormal set $\left\{p_{n}(z)\right\}$ each function of which is regular in a region $B^{\prime}$ which contains $B$ completely in its interior. For instance, suppose that $B$ is such that there is a complete orthonornal set of polynomials. ${ }^{4}$ Then the functionals (4.1) are defined not only for $f \in L^{2}(l)$, but for all $f$ belonging to the larger class $f=L^{1}(i ;$ of all singlevalued analytic functions, regular in $B$, for which

$$
\iint_{B}|f(z)| d x d y<\infty
$$

(2) Let $L_{n}(f)=L_{n}^{*}(f)$, where the $L_{n}^{*}$ are now the (normalized) functionals described in (2.17)-(2.18). The functionals $L_{n}^{*}(f)$ are defined over the class $S$ of functions possessing definitions at $z=\alpha_{i}$; and, in view of $(2.20), L_{n}$ has a representation of form (4.1).

(3) Indeed, selecting $\left\{L_{n}\right\}$ as any set of functionals defined over a set $S \supset L^{2}(B)$, satisfying (a) and (b), and construcing $L_{n}^{*}$ in accordance with $\$ 2$, we may then set $L_{n}=L_{n}^{*}$.

T'HEOREM 3. Let $f(z)$ be a function which is regular in B, which possesses the generalized Fourier coefficients

$$
a_{n}=L_{n}(f) \quad(n=0,1, \cdots),
$$

but which is not of class $L^{2}(B)$. Then there exists a change of sign $\epsilon_{n}, \epsilon_{n}=$ +1 or -1 , such that if

$$
a_{n}^{*}=\epsilon_{n} a_{n} \quad(n=0,1,2, \ldots)
$$

${ }^{4}$ See [5] for sufficient conditions on the boundary of $B$. 
are the generalized Fourier coefficients of a function $f^{*}(z)$ which is regular in $B$, then this latter function $f^{*}(z)$ cannot be continued analytically across any portion of the boundary of 13 .

Before giving the proof of Theorem 3, we shall make a number of reinarks as to its statement. As far as the proof of the theorem in the general form given above is concerned, it is necessary to make the hypothesis that $f$ not be of class $L^{2}(B)$. It is clear that some sort of restrictive hypothesis is necessary in order to prevent the original $f(z)$, and hence (possibly) the modified $f^{*}$, from being analytic in a larger region. For the special case in which $B$ is the unit circle, it is thus seen that the present change-of-sign theorem is weaker than the classical one, and although it has been found possible to modify the argument which follows so that it applies to a much wider class of functions than those which are not of $L^{2}(B)$, the proof of the full theorem along these lines has not been forthcoming.

The proof of the theorem in no way uses the existence of a function $f$ for which (4.2) bolds, but only the fact that

$$
\sum_{n=0}^{\infty}\left|a_{n}\right|^{2}=\infty \text {. }
$$

However, even if the numbers $\left\{a_{n}\right\}$ are the generalized Fourier coefficients of a function which is regular in $B$, it is by no means clear that the numbers $\left\{\epsilon_{n} a_{n}\right\}$ are again the Fourier coefficients of a second function $f^{*}$ regular in $B$. For this reason the hypothesis has been introduced into the statement of the theorem. Indeed, this property would seem to depend in part upon the choice of the complete orthonormal system $\left\{p_{n}(z)\right\}$. But we can show that for all B bounded by a finite number of Jordan curves there are complete orthonormal systems for which this will be true. One important case is that in which the functionals $L_{n}$ are the $L_{n}^{*}$ given by (2.16), (2.17). By the discussion following (2.17), it is clear that if $f$ is regular in $B$ then

$$
\limsup _{n \rightarrow \infty}\left|L_{n}^{*}(f)\right|^{1 / n} \leq 1
$$

and

$$
\limsup _{n \rightarrow \infty}\left|\epsilon_{n} L_{n}^{*}(f)^{1 / n}\right| \leq 1
$$

and so there exists a function $f^{*}$ regular in $B$ such that 


$$
\epsilon_{n} L_{n}^{*}(f)=L_{n}^{*}\left(f^{*}\right) \quad(n=0,1, \cdots)
$$

We turn now to the proof of Theorem 3. Let $\left\{C_{n}\right\}$ be the family of regions satisfying (c) and (d) above whose existence has been assumed. Let us fix our attention upon a particular region $C_{j}$. Since $B \subset C_{j}$, we get $L^{2}\left(C_{j}\right) \subset L^{2}(B)$, and so each functional $L_{n}$ of the set $\left\{L_{n}\right\}$ is defined over a class $S$ wider than $L^{2}\left(C_{j}\right)$. Let us now verify briefly that the conditions of Theorem 2 hold for the set $\left\{L_{n}\right\}$. ( The region $B$ of Theorem 2 is now to be identified with the present region $C_{j}$.) We need only show that each $L_{n}$ is bounded over $L^{2}\left(C_{j}\right)$. This is clear, for by $(4.1)$ and for $f \in L^{2}\left(C_{j}\right)$ we have

$$
L_{n}(f)=\iint_{B} f \overline{p_{n}} d x d y,
$$

so that

$$
\left|L_{n}(f)\right|^{2} \leq \iint_{B}|f|^{2} d x d y \leq \iint_{C_{j}}|f|^{2} d x d y .
$$

By Theorem 2 and the remarks following it, we know that there exist functionals

$$
L_{n}^{*(j)}=\sum_{k=0}^{n} a_{n k}^{(j)} L_{k}
$$

such that if for some $g \in S$ we have

$$
\sum_{n=0}^{\infty}\left|L_{n}^{*(j)}(g)\right|^{2}=\infty
$$

then it must follow that $g \notin L^{2}\left(C_{j}\right)$. If, therefore, for a given $g(z)$ which is regular in $B$ we have

$$
\sum_{n=0}^{\infty}\left|L_{n}^{*(j)}(g)\right|^{2}=\infty \quad(j=0,1,2, \ldots)
$$

then, as we previously have seen, by the very selection of the regions $\left\{C_{j}\right\}$, it follows that $B$ is the complete region of existence of $g$.

Let there now be given an $f(z)$ which is regular in $B$, possessing the generalized Fourier coefficients $a_{n}=L_{n}(f)$, and not of class $L^{2}(B)$. This last state- 
ment when expressed in terms of $L_{n}(f)$ means, in virtue of ( 4.1$)$, that

$$
\sum_{n=0}^{\infty}\left|a_{n}\right|^{2}=\sum_{n=0}^{\infty}\left|L_{n}(f)\right|^{2}=\infty
$$

We shall show how a change of $\operatorname{sign}\left\{\epsilon_{n}\right\}$ may be selected such that

$$
\sum_{n=0}^{\infty}\left|\sum_{k=0}^{n} a_{n k}^{(j)} \epsilon_{k} L_{k}(f)\right|^{2}=\infty \quad(j=0,1, \ldots) \text {. }
$$

Assuming now that

$$
\epsilon_{k} L_{k}(f)=\dot{L}_{k}\left(f^{*}\right)
$$

for some function $f^{*}$ regular in $B$, then, by what we have just said, the function $f^{*}$ must possess $B$ as its entire region of existence.

In order to show how a change of sign $\left\{\epsilon_{n}\right\}$ for which $(4.10)$ holds may be selected, we require two preliminary observations. The first is as follows. Let there be given two arbitrary complex numbers $z_{1}, z_{2}$, then for some change of $\operatorname{sign} \epsilon= \pm 1$ we have

$$
\left|z_{1}+\epsilon z_{2}\right| \geq\left|z_{2}\right| \text {. }
$$

For it is evident geometrically that if $\left|z_{1}+z_{2}\right|<\left|z_{2}\right|$ then $\left|z_{1}-z_{2}\right|>\left|z_{2}\right|$. Secondly, it will be important to give an estimate of the quantity $a_{n n}^{(j)}$. From (2.8a) and (2.9), it appears that

$$
\left.a_{n n}^{(j)}=l_{0}^{(j)} l_{1}^{(j)} \ldots l_{n}^{j}\right) \vdots_{0}\left(b_{i}^{*(j)}\right) L_{1}\left(\delta_{1}^{*(j)}\right) \ldots L_{n-1}\left(\phi_{n-1}^{*(j)}\right),
$$

so that, by (2.6),

$$
a_{n n}^{(j)}=\frac{1}{L_{n}\left(\phi_{n}^{*(j)}\right)} . \quad(n=0,1, \ldots, j=0,1, \ldots) .
$$

Now the functions $\phi_{n}^{*}(j)$ are of class $L^{2}\left(C_{j}\right)$ and a fortiori of class $L^{2}(B)$. Hence, by (4.1),

$$
L_{n}\left(\phi_{n}^{*(j)}\right)=\iint_{B} \phi_{n}^{*(j)} \overline{p_{n}} d x d y,
$$

so that, by the Schwarz inequality, 


$$
\left|L_{n}\left(\phi_{n}^{*(j)}\right)\right|^{2} \leq \iint_{B}\left|p_{n}\right|^{2} d x d y \iint_{B}\left|\phi_{n}^{*(j)}\right|^{2} d x d y \leq \iint_{C_{j}}\left|\phi_{n}^{*(j)}\right|^{2} d x d y=1
$$

because of the normality of the function $p_{n}$ over $B$ and the $\phi_{n}^{*(j)}$ over $C_{j}$. Thus we have

$$
\left|a_{n n}^{(j)}\right| \geq 1
$$

for all $n$ and $j$.

We return now to the construction of the sequence $\left\{\epsilon_{n}\right\}$. First select a sequence of integers $n_{0}=0, n_{1}, n_{2}, \cdots$, such that

$$
\sum_{n=n_{k}}^{n=n_{k}+1}\left|L_{n}(f)\right|^{2} \geqq 1 \quad(k=0,1, \ldots) .
$$

This is clearly possible because of (4.9). We shall next show how to select a change of $\operatorname{sign} \epsilon_{0}, \epsilon_{1}, \cdots, \epsilon_{n_{1}}$ so that

$$
\sum_{n=0}^{n}\left|\sum_{k=0}^{n} a_{n k}^{(0)} \epsilon_{k} L_{k}(f)\right|^{2} \geq 1
$$

To this end, select $\epsilon_{0}=1$; then by (4.16) we have

$$
\left|a_{00}^{(0)} \epsilon_{0} L_{0}(f)\right| \geq\left|L_{0}(f)\right| \text {. }
$$

Having selected $\epsilon_{0}, \epsilon_{1}, \cdots, \epsilon_{p}$ for $0 \leq p<n_{1}$, select $\epsilon_{p+1}$ as follows: let $\epsilon_{p+1}$ be that change of sign for which

$$
\begin{aligned}
\mid \sum_{k=0}^{p} a_{p+1, k}^{(0)} \epsilon_{k} L_{k}(f) & +a_{p+1, p+1}^{(0)} \epsilon_{p+1} L_{p+1}(f) \mid \\
& \geq\left|a_{p+1, p+1}^{(0)} L_{p+1}(f)\right| \geq\left|L_{p+1}(f)\right| .
\end{aligned}
$$

This is possible by our first observation above and by (4.16). We proceed in a step-by-step fashion, selecting one $\epsilon$ after another, and we finally arrive at $\epsilon_{0}$, $\epsilon_{1}, \ldots, \epsilon_{n_{1}}$ for which it can be asserted that

$$
\left|\sum_{k=0}^{p} a_{p, k}^{(0)} \epsilon_{k} L_{k}(f)\right| \geq\left|L_{p}(f)\right| \quad\left(p=0,1, \cdots, n_{1}\right) .
$$

The inequality (4.18) now follows from (4.20) and (4.17). 
By precisely the same argument, it is possible to select changes of sign $\epsilon_{n_{1}+1}, \epsilon_{n_{1}+2}, \cdots, \epsilon_{n_{2}}$ such that

$$
\left|\sum_{n=n_{1}+1}^{n_{2}} \sum_{k=0}^{n} a_{n k}^{(1)} \epsilon_{k} L_{k}(f)\right|^{2} \geq 1 .
$$

For we may select $\epsilon_{n_{1}+1}$ as that change of sign for which

$$
\begin{aligned}
& \left|\sum_{k=0}^{n_{1}} a_{n_{1}+1, k}^{(1)} \epsilon_{k} L_{k}(f)+a_{n_{1}+1, n_{1}+1}^{(1)} \epsilon_{n_{1}+1} L_{n_{1}+1}(f)\right| \\
& \quad \geq\left|a_{n_{1}+1, n_{1}+1}^{(1)} L_{n_{1}+1}(f)\right|
\end{aligned}
$$

the rest of the argument proceeding as before. We now proceed through the sequence of matrices $T^{(j)}=\left(a_{n k}^{(j)}\right)$ in an oscillatory fashion, returning to each matrix an infinite number of times. To formalize this process, let us associate with each matrix $T^{(j)}$ an infinite number of intervals of integers $\left(n_{p}+1 \leq n \leq\right.$ $\left.n_{p+1}\right)$ in the following way:

$$
\begin{aligned}
& T_{0}: \quad\left(n_{0}, n_{1}\right) \quad\left(n_{2}+1, n_{3}\right) \quad\left(n_{5}+1, n_{6}\right) \quad \ldots \\
& T_{1}:\left(n_{1}+1, n_{2}\right) \quad\left(n_{4}+1, n_{5}\right) \\
& T_{2}:\left(n_{3}+1, n_{4}\right)
\end{aligned}
$$

Thus to each matrix $T^{(j)}$ we have associated an infinite number of distinct intervals

$$
\left(n_{p_{k}}^{(j)}+1, n_{p_{k}^{(j)}+1}\right) \quad(k=0,1,2, \cdots) .
$$

Conversely, to each integer $q \geq 0$ there is associated a unique integer $j=j(q)$ such that the interval $\left(n_{q}+1, n_{q+1}\right)$ is associated with the matrix $T^{(j(q))}$. Having now selected changes of $\operatorname{sign} \epsilon_{0}, \epsilon_{1}, \cdots, \epsilon_{n_{q}}$, we proceed to select changes of sign $\epsilon_{n_{q}+1}, \epsilon_{n_{q}+2}, \cdots, \epsilon_{n_{q+1}}$ in such a way that

$$
\sum_{n=n_{q}+1}^{n_{q+1}}\left|\sum_{k=0}^{n} a_{n k}^{(j)} \epsilon_{k} L_{k}(f)\right|^{2} \geq 1 \quad(j=j(q)) .
$$


This can be done for $q=0,1, \ldots$.

Let now $j$ be any fixed integer $\geqq 0$, and let

$$
\left(n_{p_{r}^{(j)}}+1, n_{p_{r}^{(j)}+1}\right) \quad(r=0,1,2, \cdots)
$$

be the intervals associated with $T^{(j)}$. Then

$$
\sum_{n=0}^{\infty}\left|\sum_{k=0}^{n} a_{n k}^{(j)} \epsilon_{k} L_{k}(f)\right|^{2} \geq \sum_{r=0}^{\infty}\left|\sum_{n=\left(n_{p r}^{(j)}\right)+1}^{n=n} a_{n k}^{(j)} \epsilon_{k}^{(j)} L_{k}(f)\right|^{2}=\infty
$$

The first inequality follows from the fact that the left-hand memier includes all the terms of the middle number, while the divergence follows from (4.23). Thus, our selection of $\left\{\epsilon_{k}\right\}$ has guaranteed the inequality (4.10), and the theorem is established.

3. Gap theoreins. It is instructive to apply the method of The orem 3 to derive gap theorems for series of orthogonal functions inasmuch as this method exhibits clearly the role played by the deletion of coefficients. The theorem obtained by this means assures the existence of gaps leading to natural boundaries, but gives no information as to their density. These results are therefore of interest for the case of general regions ${ }^{5}$ and for general functionals, but of little interest for the case of power series in the unit circle which possesses a highly developed theory of gaps. In the present section, we retain all the assumptions of $\S 4$, regarding the region $B$ and the set of functionals $\left\{L_{n}\right\}$. We assume moreover that there is a fixed larger closed domain $\bar{B}^{\prime}$ containing $B$ in its interior such that each function $p_{n}(z)$ of the orthonormal set in $(4.1)$ is regular in $\bar{B}^{\prime}$.

THEOREM 4. Let $\left\{\alpha_{n}\right\}$ be a sequence which is bounded away from zero:

$$
\left|\alpha_{n}\right| \geq \alpha>0 \quad(n=0,1, \cdots) .
$$

There exists a sequence $\left\{\delta_{n}\right\}, \delta_{n}=0$ or 1 , such that if $i_{n} \alpha_{n}$ are the (generalized) Fourier coefficients (with respect to the system $\left\{p_{n}(z)\right\}$ ) of a function $f$ which is regular in $B$ :

$$
\hat{o}_{n} \alpha_{n}=i_{n}(r)
$$

then $f$ posses ses the boundary of $B$ as a natural boundary.

\footnotetext{
${ }^{5} \mathrm{Cf}$. [10] where a related subject is investigated from a different point of view.
} 
Proof. We may evidently select the set of regions $C^{(j)}$ so that $C^{(j)} \subset B^{\prime}$ $(j=0,1, \cdots)$. Each function $p_{k}(z)$ is of class $L^{2}\left(B^{\prime}\right)$ and a fortiori of class $L^{2}\left(C^{(j)}\right)$. Thus we have

$$
\sum_{n=0}^{\infty}\left|\sum_{m=0}^{n} a_{n m}^{(j)} L_{m}\left(p_{k}(z)\right)\right|^{2}<\infty \quad(k=0,1, \cdots, j=0,1, \cdots),
$$

so that, in view of the orthonormality of $\left\{p_{k}\right\}$ over $B$, we have

$$
\sum_{n=k}^{\infty}\left|a_{n k}^{(j)}\right|^{2}<\infty \quad(k=0,1, \cdots, j=0,1, \ldots) .
$$

This means in particular that, for fixed $j$ and $k$, we have

$$
\lim _{n \rightarrow \infty} a_{n k}^{(j)}=0 .
$$

As before, we have

$$
\left|a_{n n}^{(j)}\right| \geq 1 \quad(n, j=0,1,2, \cdots) .
$$

We shall show that we can find a row in the zero-th matrix $T^{(0)}$ with index $r_{1}$, and numbers $\delta_{n}=0$ or $1\left(n=0,1, \cdots, r_{1}\right)$, such that

$$
\left|\sum_{k=0}^{r_{1}} a_{r_{1} k}^{(0)} \delta_{k} \alpha_{k}\right| \geq \frac{\alpha}{2}
$$

This is clear; for we may select $r_{1}=0, \epsilon_{0}=1$, and (5.7) follows in view of (5.6) and (5.1). We now use the oscillatory process of selection which was employed in the proof of Theorem 3. Assume that we have selected rows $r_{1}$ in $T^{(0)}, r_{2}$ in $T^{(1)}, r_{3}$ in $T^{(0)}, r_{4}$ in $T^{(1)}, r_{5}$ in $T^{(2)}, r_{6}$ in $T^{(0)}, \cdots, r_{q}$ in $T^{(t)}$, $t=t(q)$, and numbers $\delta_{n}(=0$ or 1$)\left(n=0,1, \cdots, r_{q}\right)$ such that

$$
\left|\sum_{k=0}^{r_{p}} a_{r_{p}, k}^{(t)} \delta_{k} a_{k}\right| \geq \frac{\alpha}{2}, \quad(t=t(p) ; p=0,1, \ldots, q) .
$$

We shall now show how to select a row $r_{q+1}$ in the matrix $T^{(t(q+1))}$ and numbers $\delta_{n}\left(r_{q}+1 \leq n \leq r_{q+1}\right), \delta_{n}=0$ or 1 , such that

$$
\left|\sum_{k=0}^{r_{q+1}} a_{r_{q+1}, k}^{t(q+1)} \delta_{k} \quad a_{k}\right| \geq \frac{\alpha}{2}
$$


In the matrix $T^{t(q+1)}$, we consider the first $r_{q}$ columns. By (5.5), we have,

$$
\lim _{n \rightarrow \infty} a_{n k}^{t(q+1)}=0 \quad\left(k=0,1, \cdots, r_{q}\right) ;
$$

hence, defining a quantity $\eta$ by

$$
\eta=\frac{\alpha}{2} \sum_{k=0}^{r_{q}}\left|\alpha_{k}\right|,
$$

we may select (by (5.5)) $n$ so large that

$$
\left|a_{n k}^{t(q+1)}\right| \leq \eta \quad\left(k=0,1, \cdots, r_{q}\right) .
$$

Designate the $n$ chosen in this manner by $r_{q+1}$, and select $\delta_{n}=0$ for $r_{q}+1 \leq n \leq$ $r_{q+1}-1$, while $\delta_{n}=1$ for $n=r_{q+1}$. Thus, the sum in (5.9) becomes

$$
\begin{aligned}
\left|\sum_{k=0}^{r_{q+1}} a_{r_{q+1}, k}^{t(q+1)} \delta_{k} \alpha_{k}\right| & =\left|\sum_{k=0}^{r_{q}} a_{r_{q+1}, k}^{t(q+1)} \delta_{k} \alpha_{k}+a_{r_{q+1}, r_{q+1}}^{t(q+1)} \delta_{r_{q+1}} a_{r_{q+1}}\right| \\
& =|A+B| \geq|| B|-| A||,
\end{aligned}
$$

where $A$ refers to the sum appearing in the middle of (5.12), while $B$ designates the isolated term. From ( 5.11 ) we have

$$
|A| \leq \eta \cdot \sum_{k=0}^{r_{q}}\left|\alpha_{k}\right|=\frac{\alpha}{2},
$$

while from (5.6) and (5.1) we get

$$
|B| \geq \alpha,
$$

so that $(5.9)$ holds. For each fixed matrix $T^{(j)}$ we have an infinite number of inequalities of type (5.9) corresponding to an infinite number of its rows. Thus clearly,

$$
\begin{aligned}
& \sum_{n=0}^{\infty} \mid \sum_{k=0}^{n} a_{n k}^{(j)} \delta_{k}\left.\alpha_{k}\right|^{2}=\sum_{n=0}^{\infty}\left|\sum_{k=0}^{n} a_{n k}^{(j)} L_{k}(f)\right|^{2}=\infty \\
&(j=0,1,2, \ldots),
\end{aligned}
$$

and our theorem is proved. Again we should observe that under conditions (5.1) 
we have no assurance a priori that $\left\{\delta_{n} \alpha_{n}\right\}$ are the generalized Fourier coefficients of a function which is regular in $B$. But the example mentioned in connection with Theorem 3 shows that for all $B$ bounded by a finite number of Jordan curves, there are complete orthonormal systems for which this is the case.

\section{References}

1. S. Bergman, Zur Theorie der linearen Integral-und Funktionalgleichungen im komplexen Gebiet, Bull. [Izvestya] Math. Mech. Inst. Univ. Tomsk. 1 (1935-37), 242-257.

2. - The kernel function and conformal mapping, Mathematical Surveys No.5, American Mathematical Society, New York, 1950.

3. L. Bieberbach, Lehrbuch der Funktionentheorie, vol. II, Leipzig-Berlin, 1927.

4. P. Davis, On the applicability of linear differential operators of infinite order to functions of the class $L^{2}(B)$, Amer. J. Math. 74 (1952), 475-491.

5. O. J. Farrell, On approximation to an analytic function by polynomials, Bull. Amer. Math. Soc. 40 (1934), 908-914. 400.

6. P. Fatou, Séries trigonométriques et séries de Taylor, Acta Math. 30 (1906), 335-

7. A. Hurwitz and G. Pólya, Zwei Beweise eines von Herrn Fatou vermuteten Satzes, Acta Math. 40 (1916), 180.

8. G. Pólya, Über die Potenzreihen deren Konvergenzkreis naẗ̈̈iiche Grenze ist, Acia Math. 41 (1916), 180.

9. J. L. Walsh, Interpolation and approximation, Amer. Math. Soc. Colloquium Publ., vol. 20, New York, 1935.

10. - Overconvergence, degree of convergence, and zeros of sequences of analytic functions, Duke Math. J. 13 (1946), 195-234.

11. J. L. Walsh and P. Davis, Interpolation and orthonormal systems, J. d'Analyse Math. (To appear).

American University, Washington, D. C.

The Bell Telephone laboratories, Murray Hill, N. J. 


\section{PACIFIC JOURNAL OF MATHEMATICS}

\section{EDITORS}

\author{
R. M. Foeinson \\ University of California \\ Berkeley 4, California \\ E. HEWITt \\ University of Washington \\ Seattle 5, Washington
}

R. P. DILWOR TH

California Institute of Technology

Pasadena 4, California

E. F. BECKENBACH

University of California

Los Angeles 24, California

\section{ASSOCIATE EDITORS}

$\begin{array}{llll}\text { H. BUSEMANN } & \text { P. R. HALMOS } & \text { BøRGE JESSEN } & \text { J. J. STOKER } \\ \text { HERBERT FFDERER } & \text { HEINZ HOPF } & \text { PAUL LÉVY } & \text { E. G. STR AUS } \\ \text { MARSHALL IIALI } & \text { R. D. JAMES } & \text { GEORGE PÓLYA } & \text { KỎSAKU YOSIDA }\end{array}$

\section{SPONSORS}

UNIVERSITY OF BRITISH COLUMBIA CAIIFORNIA INSTITUTE OF TECHNOLOGY UNIVERSITY OF CALIFORNIA, BERKELEY UNIVERSITY OF CAILIFORNIA, DAVIS UNIVERSITY OF CALIFORNIA, LOS ANGELES UNIVERSITY OF CALIFORNIA, SANTA BARBARA UNIVERSITY OF NEVADA OREGON STATE COLLEGE UNIVERSITY OF OREGON
UNIVERSITY OF SOUTHERN CALIFORNIA STANFORD RESEARCH INSTITUTE STANFORD UNIVERSITY WASHINGTON STATE COLLEGE UNIVERSITY OF WASHINGTON

AMERICAN MATHEMATICAL SOCIETY NATIONAL BUREAU OF STANDARDS, INSTITUTE FOR NUMERICAL ANALYSIS

$$
\begin{gathered}
\text { Vari-Type Composition by } \\
\text { Elaine Barth } \\
\text { Delores Wierman } \\
\text { With the cooperation of } \\
\text { E. F. Beckenbach } \\
\text { E. G. Straus }
\end{gathered}
$$

Printed in the United States of America by Edwards Brothers, Inc., Ann Arbor, Michigan 


\section{Pacific Journal of Mathematics}

\section{Vol. 3, No. 1 \\ March, 1953}

Herbert Busemann, Volume in terms of concurrent cross-sections ......... 1

L. Carlitz, Some special equations in a finite field ................. 13

Homer V. Craig and Billie Braden Townsend, On certain metric

extensors ....................................... 25

Philip J. Davis and Henry Pollak, Linear functionals and analytic

continuation problems ............................. 47

Jacob C. E. Dekker, The constructivity of maximal dual ideals in certain

Boolean algebras ................................. 73

Harley M. Flanders, The norm function of an algebraic field extension .... 103

Marshall Hall, Subgroups of free products . . . . . . . . . . . . . . . . . 115

Israel (Yitzchak) Nathan Herstein, Finite multiplicative subgroups in

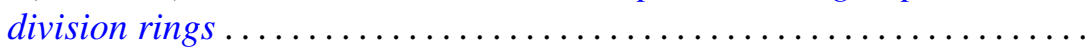

Joseph Lawson Hodges, Jr. and Murray Rosenblatt, Recurrence-time moments in random walks ............................ 127

Alfred Horn, The normal completion of a subset of a complete lattice and lattices of continuous functions ........................ 137

Fulton Koehler, Estimates for the errors in the Rayleigh-Ritz method...... 153

M. H. Martin, The Monge-Ampère partial differential equation

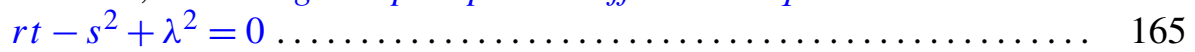

John E. Maxfield, Normal k-tuples ......................... 189

Jack E. McLaughlin, Structured theorems for relatively complemented lattices............................................ 197

William H. Mills, A system of quadratic Diophantine equations ......... 209

T. S. Motzkin, Ernst Gabor Straus and F. A. Valentine, The number of farthest points ................................. 221

G. Power, Forces on the boundary of a dielectric ............... 233

Ralph Gordon Selfridge, Approximations with least maximum error....... 247 